PUBLICATIONS OF THE

ASTRONOMICAL SOCIETY OF THE PACIFIC

\begin{tabular}{lcc}
\hline \hline Vol. 102 & November 1990 & No. 657 \\
\hline \hline
\end{tabular}

\title{
SPECTROSCOPY OF RADIO SOURCES FROM THE PARKES 2700 MHz SURVEY
}

\author{
D. J. THOMPSON, S. DJORGOVSKI, ${ }^{*} †$ AND R. DE CARVALHO $\$$ \\ Palomar Observatory, California Institute of Technology, Pasadena, California 91125 \\ Received 1990 June 28 , revised 1990 July 30
}

\begin{abstract}
We present the results of long-slit CCD spectroscopy on 37 objects from the Parkes $2700 \mathrm{MHz}$ survey, with data for an additional two companion objects. Eight of the objects are quasars, six more are AGNs, and five more are radio galaxies. Seventeen of the objects observed are stars and, thus, probable misidentifications. Three objects show featureless spectra and are identified as possible BL Lac objects. Spectra are presented for a total of 20 objects.
\end{abstract}

Key words: galaxies: redshifts-quasars-redshifts-spectroscopy

\section{Introduction and Observations}

The Parkes (PKS) $2700 \mathrm{MHz}$ survey is a complete survey for objects with a limiting flux density of approximately $S_{2700} \geq 0.25 \mathrm{Jy}$ (Bolton et al. 1975; Bolton, Savage, and Wright 1979; and references therein). We have undertaken a follow-up slit spectroscopy program at medium resolution ( $3 \AA-6 \AA$ ) of the objects in the PKS survey, using no systematic selection criteria, as a marginal weather backup project. In general, objects were selected on the basis of optical brightness or possibly interesting morphology on the published finding charts. Our goal is to classify the objects, obtain accurate redshifts, and identify objects worth pursuing in more detail.

The observations were done on UT 1988 April 8-10, UT 1988 June 8-9, UT 1988 September 12, and UT 1989 November 27 with the Modular Spectrograph on the 100-inch (2.5-m) telescope at Las Campanas; on UT 1988 July 14-16 with the 4-meter telescope at Cerro Tololo; and on UT 1988 January 10 and UT 1988 March 11 with the Double Spectrograph (Oke and Gunn 1982) at the Cassegrain focus of the Palomar Observatory Hale 200inch (5-m) telescope. The weather was generally nonphotometric, with variable transparency due to cirrus. The

\footnotetext{
*Alfred P. Sloan Foundation fellow.

†Visiting Astronomer, Cerro Tololo Inter-American Observatory, National Optical Astronomy Observatories, operated by AURA, Inc., under cooperative agreement with the National Science Foundation.

$\ddagger$ Visiting Investigator, Las Campanas Observatory, Carnegie Institution of Washington.

§Permanent address: Observatorio Nacional-SPNq, Rua Gal. Bruce 586, São Cristóvão, Rio de Janeiro, Brazil.
}

seeing ranged from 1.5 to 3 arc seconds (FWHM). The slit widths and gratings varied but were chosen to give 3 to 6 angstrom resolution and covering approximate ranges of 4300 to 7300 angstroms. Exposure times ranged from 400 to 2000 seconds.

Wavelength calibration was accomplished with helium, neon, argon, and hollow cathode (Fe-Ar) lamps, giving an rms error in the dispersion solutions of $\leq 1 \AA$. Wavelength shifts due to instrument flexure were compensated for by taking frequent spectra of the helium arc lamps throughout the night or by extracting a sky spectrum adjacent to each object. Dome flat fields and spectroscopic flux standards from Oke and Gunn (1983), Stone (1977), Stone and Baldwin (1983), and Baldwin and Stone (1984) were used to correct for instrument response and to give an approximate flux calibration. Due to the variable transparency, the zero-point uncertainties of the flux calibration are estimated to be on the order of 0.5-1.0 magnitude. The data were reduced using standard procedures.

Thirty-seven PKS objects were observed, of which eight are quasars and six are Seyferts or active galaxies. An additional five objects are radio galaxies, only one of which shows weak emission lines. Three objects could not be identified due to the absence of any identifiable spectral features, two of which were previously noted as having featureless continuum spectra (0118-272 and $0823-223)$. We find some evidence for variability in 0118-272 and 1424+240, supporting the interpretation that these objects are BL Lacertaes. In Table 1 we list the emission-line data derived from the spectra of the quasars 
and strong emission-line galaxies, while in Table 2 we list the same information for the absorption-line radio galaxies and "featureless spectrum" objects in this sample. None of these objects were found in the IRAS PSC. Seventeen PKS objects were found to be stars and are thus probable misidentifications of the radio sources. These are listed in Table 3. Figure 1 shows the spectra of all the nonstellar Parkes objects, not including the possible BL Lacs.

\section{Comments on Selected Objects}

PKS 0035-392: A 16.5 quasar at $z=0.596$ with a companion stellar object $\sim 4$ arc sec away in P.A. $=155^{\circ}$. We find that the companion is an M-type star. The spectrum of the quasar is remarkably similar to that of 3C 48 published by Phillips (1977). This quasar was previously identified by Peterson, Jauncey, and Wright (1976), who derived a redshift of 0.592 . The excess $J, H$, and $K$ flux found by Glass (1981) is most likely due to the M star.

PKS 0118-272: This possible BL Lac object was observed previously by Peterson et al. (1976), who noted the featureless continuum at $\sim 16^{\mathrm{m}} .5$. We find a continuum magnitude of $A B_{v}=14.5$ at $\lambda=6000 \AA$, which may indicate some variability, though the spectrum was obtained under nonphotometric conditions.

PKS 0823-223: A $16^{\mathrm{m}}$ possible BL Lac object. Wright, Ables, and Allen (1983) noted the featureless continuum of this object, but our spectrum shows two absorption lines, one apparently due to Na D $\lambda 5893$, which is probably interstellar in origin, and the other at $\lambda 5349$ which remains unidentified. We find no evidence for variability.

PKS 0858-279: Although the optical counterpart to this source, previously proposed by Bolton et al. (1975), is a 14.5 galactic star, there is a $17^{\mathrm{m}}$ quasar 3 arc sec north at P.A. $=0^{\circ}$, which we identify as the radio source. There is only one emission line in the spectrum; if it is $\mathrm{Mg}$ II $\lambda 2798$, then we find $z=1.14$.

PKS 0858-771: An $18^{\mathrm{m}}$ Seyfert I at $z=0.4900$. This object was previously identified by Wilkes (1986), who found $z=0.489$.

PKS 0903-573: Although the optical counterpart proposed by White et al. (1987) is a $17^{\mathrm{m}}$ star, there is a $19^{\mathrm{m}}$ Seyfert I at $z=0.695$ that is $4 \operatorname{arc~sec}$ west at P.A. $=265^{\circ}$, which we identify as the radio source.

PKS 1340+05: A 17. 8 BLRG at $z=0.136$ as identified by Grandi (1983), which we confirm.

PKS 1342-314: The object identified in Shimmins and Bolton (1974) is a galaxy at $z=0.143$. Since it shows no evidence of emission lines, and is in a crowded field, this may be a misidentification of the radio source.

PKS 1424+240: Listed at 16.5 in Condon, Hicks, and Jauncey (1977), we find no obvious emission lines and several unidentified nonstellar absorption lines with a continuum magnitude of $15^{\mathrm{m}}$. We interpret this to be a possible BL Lac with intervening metallic absorber(s).
PKS 1437-153: We find the optical counterpart identified in Bolton et al. (1975) to be a $17^{\mathrm{m}} .5$ star. Condon et al. (1977) changed the ID to a $19^{\mathrm{m}}$ object approximately 13 arc sec northeast at P.A. $\simeq 40^{\circ}$ based on interferometer measurements. Véron-Cetty and Véron (1989) list this object in their Table 3 at $z=0.254$, but the reference quoted there is incorrect.

PKS 1444-301: A galaxy at $c z=4660 \mathrm{~km} \mathrm{~s}^{-1}$ which does not show any emission lines. It is by far the brightest object in the Parkes error circle and, thus, is probably a correct identification of the radio source.

PKS 1601-00: Also known as 4C-00.63. We find this $17^{\mathrm{m}}$ radio galaxy to be at $z=0.0825$.

PKS 1602-002: Also known as PKS 1602-001 and PKS $1602-00$. Identified as a $17^{\mathrm{m}}$ quasar at $z=1.625$ by Wills and Lynds (1978) and $A B_{1550}=18.1$ at $z=1.633$ by Wampler et al. (1984), we find a redshift of 1.64.

PKS 2153-219: A $17^{\mathrm{m}}$ LINER at $z=0.098$ possibly with high internal obscuration; the $\mathrm{H} \beta$ and [O III] $\lambda 5007$ lines are completely absent.

PKS 2358-161: An $18^{\mathrm{m}}$ quasar at $z=2.044$. This object was previously identified by Wright et al. (1983).

\section{Concluding Remarks}

The Parkes survey has long been a popular hunting ground for radio-selected quasars. These spectroscopic identifications have mainly concentrated on the brigher flat-spectrum objects, a majority of which turn out to be AGN. As the classification of the Parkes objects continues to fainter levels, more misidentifications can be expected. We find that fully $46 \%$ of the objects in our sample are probable misidentifications. Still, almost $40 \%$ of the objects in this work turned out to be active.

We are indebted to the staffs of Las Campanas Observatory, especially Oscar Duahalde, Angel Guerra, Fernando Peralta, and Alberta Zuniga; Cerro Tololo InterAmerican Observatory, especially M. Hernandez, M. Navarete, H. Tirado, and R. Venegas; and Palomar Observatory, especially J. Carrasco, J. Henning, and D. Tennant for their help during the observations. This work was supported in part by a Kingsley Foundation Fellowship (D.J.T.) and the Alfred P. Sloan Foundation and the California Institute of Technology (S.G.D.).

\section{REFERENCES}

Baldwin, J., and Stone, R. P. S. 1984, M.N.R.A.S., 206, 242.

Bolton, J. G., Savage, A., and Wright, A. E. 1979, Aust. J. Phys. Astrophys. Suppl., No. 46, 1.

Bolton, J. G., Shimmins, A. J., Wall, J. V., and Butler, P. W. 1975, Aust. J. Phys. Astrophys. Suppl., No. 34, 1.

Condon, J. J., Hicks, P. D., and Jauncey, D. L. 1977, A.J., 82, 692.

Glass, I. S. 1981, M.N.R.A.S., 194, 795

Grandi, S. A. 1983, M.N.R.A.S., 204, 691.

Hewitt, A., and Burbidge, G. 1987, Ap. J. Suppl., 63, 1.

Oke, J. B., and Gunn, J. E. 1982, Pub. A.S.P., 94, 586. 1983, Ap. J., 266, 713. 
Table 1

Quasars and Active Galaxies

\begin{tabular}{|c|c|c|c|c|c|}
\hline Source & Iine & rest & obs & $\mathbf{z}$ & ID, average $z$ \\
\hline PKS035-392 & $\begin{array}{l}\text { Mg II } \\
\text { [Ne V] } \\
\text { [O II] } \\
\text { [Ne III] } \\
\text { [Ne III] } \\
\text { H-delta } \\
\text { H-gamma }\end{array}$ & $\begin{array}{l}2798 \\
3426 \\
3727 \\
3869 \\
3968 \\
4102 \\
4341\end{array}$ & $\begin{array}{l}4468 \\
5465 \\
5950 \\
6175 \\
6332 \\
6540 \\
6929\end{array}$ & $\begin{array}{l}0.5969 \\
0.5953 \\
0.5965 \\
0.5960 \\
0.5959 \\
0.5946 \\
0.5965\end{array}$ & $\begin{array}{l}250 / N \text { galaxy } \\
z=0.596+/-0.001\end{array}$ \\
\hline PRS0452-515 & $\mathrm{Mg}$ II & 2798 & 6035 & 1.157 & $\begin{array}{l}250 \\
z=1.157+/-0.005\end{array}$ \\
\hline PKS0858-279 & $\operatorname{Mg} I I$ & 2798 & 5988 & 1.140 & $\begin{array}{l}250 \\
z=1.14+/-0.01\end{array}$ \\
\hline PKS0858-771 & $\begin{array}{l}\text { [No V] } \\
\text { [O II] } \\
\text { [No III] } \\
\text { [No III] } \\
\text { H-dolta } \\
\text { H-gamma } \\
\text { [O III] } \\
\text { Ho II }\end{array}$ & $\begin{array}{l}3426 \\
3727 \\
3869 \\
3968 \\
4102 \\
4341 \\
4363 \\
4686\end{array}$ & $\begin{array}{l}5103 \\
5557 \\
5764 \\
5914 \\
6110 \\
6468 \\
6502 \\
6978\end{array}$ & $\begin{array}{l}0.4895 \\
0.4911 \\
0.4898 \\
0.4905 \\
0.4895 \\
0.4902 \\
0.4901 \\
0.4891\end{array}$ & $\begin{array}{l}\text { Seyfert I } \\
z=0.4900+/-0.0005\end{array}$ \\
\hline PKS0903-573 & $\begin{array}{ll}M g & I I \\
{[0} & I I]\end{array}$ & $\begin{array}{l}2798 \\
3727\end{array}$ & $\begin{array}{l}4751 \\
6306\end{array}$ & $\begin{array}{l}0.698 \\
0.692\end{array}$ & $\begin{array}{l}\text { Seyfert I } \\
z=0.695+/-0 / 003\end{array}$ \\
\hline PKS1329-049 & $\begin{array}{l}\text { C IV } \\
\text { C III] }\end{array}$ & $\begin{array}{l}1549 \\
1909\end{array}$ & $\begin{array}{l}4792 \\
5912\end{array}$ & $\begin{array}{l}2.209 \\
2.097\end{array}$ & $\begin{array}{l}\text { Qso } \\
z=2.15+/-0.03\end{array}$ \\
\hline PRS1340+05 & $\begin{array}{l}{\left[\begin{array}{ll}0 & \text { III }\end{array}\right]} \\
{\left[\begin{array}{ll}0 & I\end{array}\right]} \\
\text { Ha } \\
{[N \quad I I]}\end{array}$ & $\begin{array}{l}5007 \\
6300 \\
6563 \\
6583\end{array}$ & $\begin{array}{l}5694 \\
7158 \\
7457 \\
7477\end{array}$ & $\begin{array}{l}0.137 \\
0.136 \\
0.136 \\
0.136\end{array}$ & $\begin{array}{l}\text { Seyfert II } \\
z=0.136+/-0.001\end{array}$ \\
\hline PRS1510-08 & $\begin{array}{l}\text { H-epsilon } \\
\text { H-dolta } \\
\text { H-gamma } \\
\text { H-beta } \\
\text { [O III] }\end{array}$ & $\begin{array}{l}3970 \\
4102 \\
4341 \\
4861 \\
5007\end{array}$ & $\begin{array}{l}5398 \\
5588 \\
5907 \\
6611 \\
6793\end{array}$ & $\begin{array}{l}0.360 \\
0.362 \\
0.361 \\
0.360 \\
0.357\end{array}$ & $\begin{array}{l}\text { Seyfert I } \\
z=0.360+/-0.002\end{array}$ \\
\hline PKS1602-002 & $\begin{array}{l}\text { C III] } \\
\mathrm{Mg} \text { II }\end{array}$ & $\begin{array}{l}1909 \\
2798\end{array}$ & $\begin{array}{l}5018 \\
7398\end{array}$ & $\begin{array}{l}1.629 \\
1.644\end{array}$ & $\begin{array}{l}\text { QSO } \\
z=1.64+/-0.01\end{array}$ \\
\hline PKS1619-680 & Mg II & 2798 & 6601 & 1.359 & $\begin{array}{l}\text { QSO } \\
z=1.36+/-0.01\end{array}$ \\
\hline PKS1814-63 & 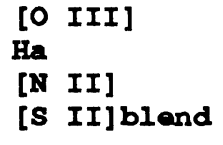 & $\begin{array}{l}5007 \\
6563 \\
6583 \\
6716\end{array}$ & $\begin{array}{l}5331 \\
6993 \\
7005 \\
7152\end{array}$ & $\begin{array}{l}0.065 \\
0.066 \\
0.064 \\
0.065\end{array}$ & $\begin{array}{l}\text { Seyfert II } \\
z=0.065+/-0.001\end{array}$ \\
\hline PKS2153-219 & $\begin{array}{l}\text { Ha } \\
\text { [S II]blend }\end{array}$ & $\begin{array}{l}6563 \\
6716\end{array}$ & $\begin{array}{l}7206 \\
7377\end{array}$ & $\begin{array}{l}0.098 \\
0.098\end{array}$ & $\begin{array}{l}\text { LINFER } \\
z=0.098+/-0.001\end{array}$ \\
\hline PKS2348-252 & $\mathrm{Mg} I I ?$ & 2798 & 6698 & 1.394 & $\begin{array}{l}\text { QSO } \\
z=1.39+/-0.01\end{array}$ \\
\hline PKS2358-161 & $\begin{array}{l}\text { C IV } \\
\text { C III] }\end{array}$ & $\begin{array}{l}1549 \\
1909\end{array}$ & $\begin{array}{l}4714 \\
5815\end{array}$ & $\begin{array}{l}2.042 \\
2.046\end{array}$ & $\begin{array}{l}\text { QSO } \\
z=2.044+1-0.002\end{array}$ \\
\hline
\end{tabular}



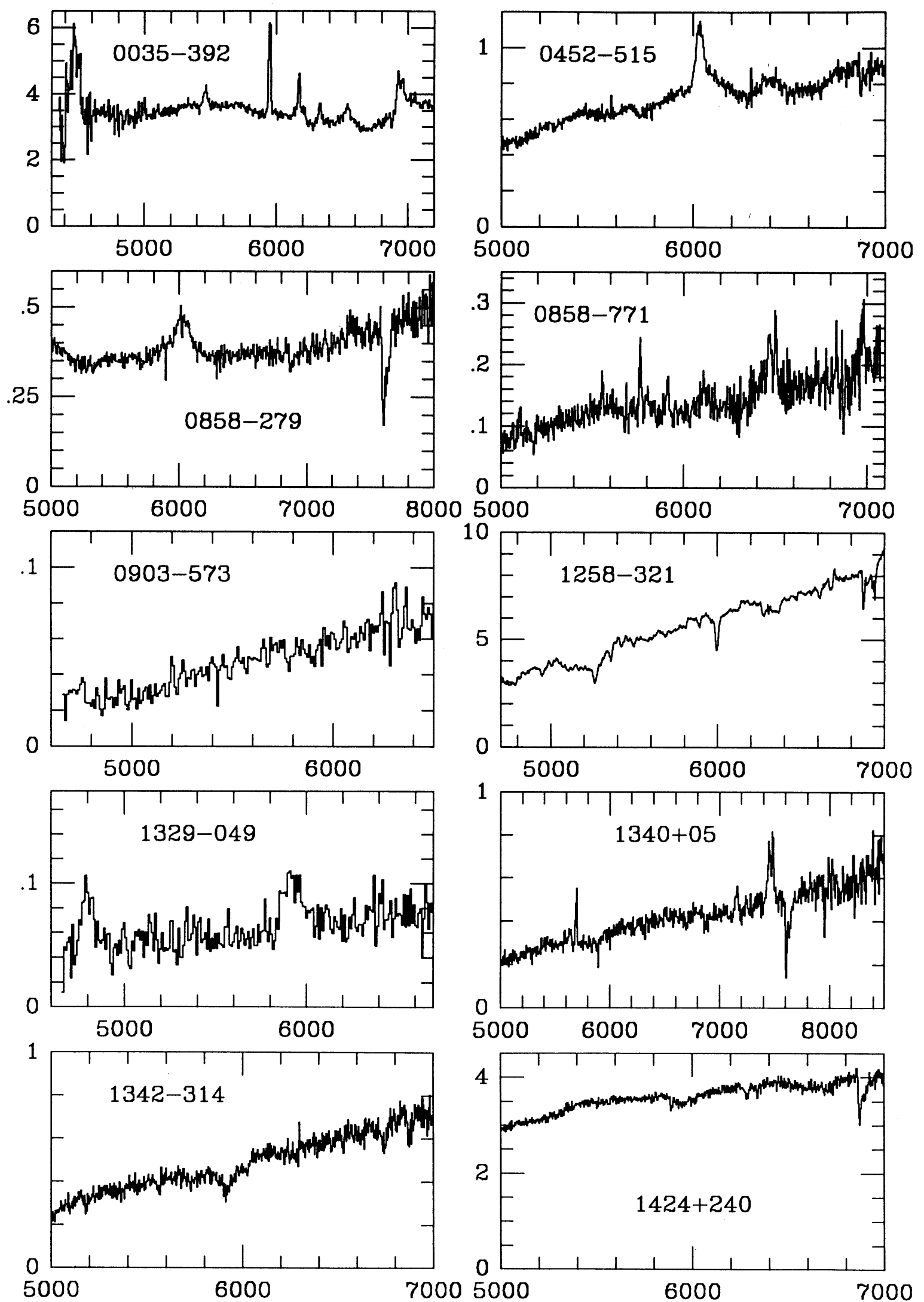

FIG. 1a-Spectra of the AGN, active galaxies, and radio galaxies in this sample. The flux calibration zero point may be uncertain by as much as $0.5-1.0$ magnitude. Spectra are plotted as $F_{v}(\mathrm{mJy})$ versus $\lambda(\AA)$. See the text for details on selected objects. 

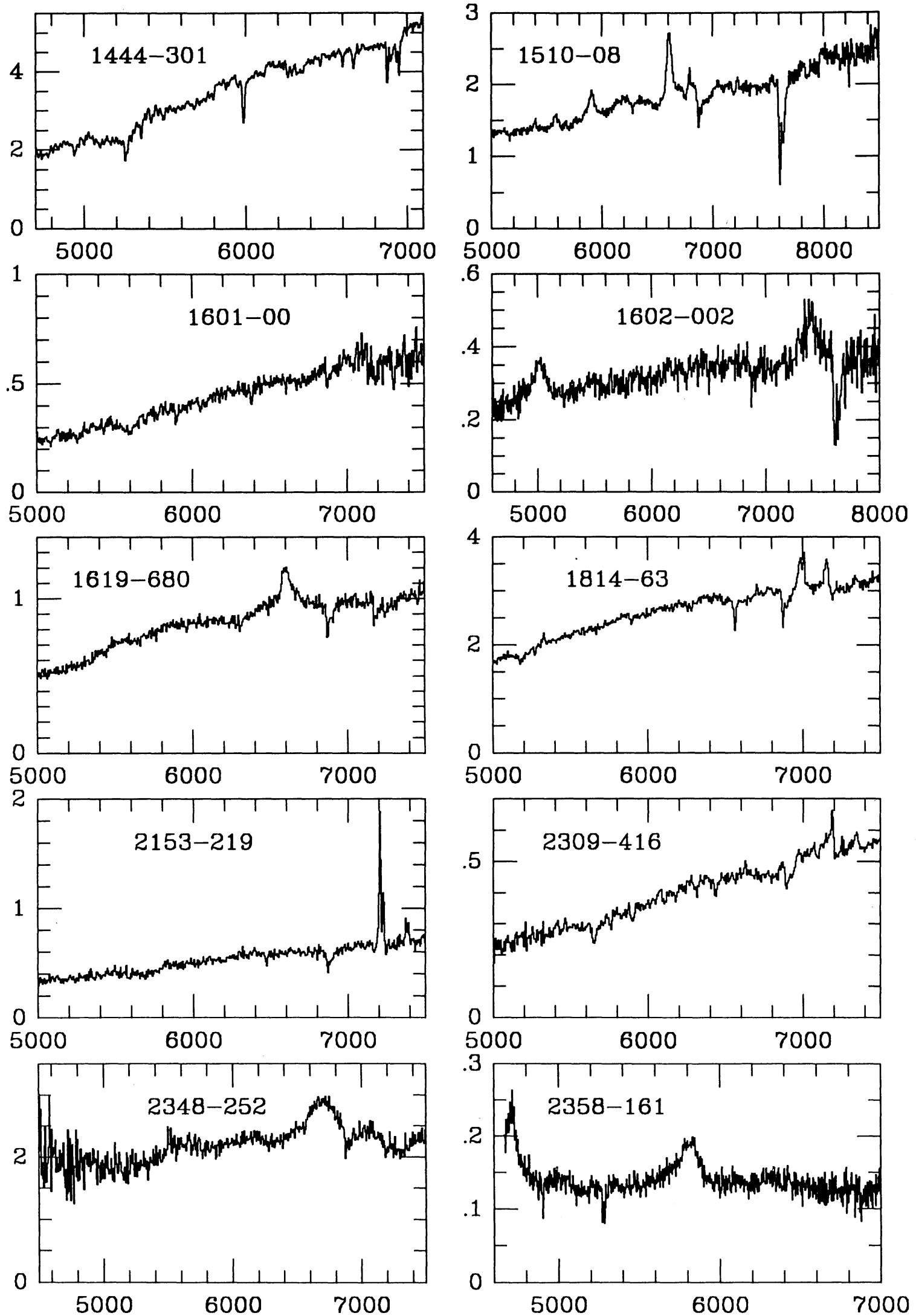

FIG. 1b-Spectra of the AGN, active galaxies, and radio galaxies in this sample. The flux calibration zero point may be uncertain by as much as $0.5-1.0$ magnitude. Spectra are plotted as $F_{v}(\mathrm{mJy})$ versus $\lambda(\AA)$. See the text for details on selected objects. 
Table 2

Radio Galaxies and Possible BL Lac Objects

\begin{tabular}{|c|c|c|c|c|c|}
\hline Source & Iine & rest & obs & $\mathbf{z}$ & ID, average $z$ \\
\hline PKS0118-272 & none & & & & Possible BI Iac \\
\hline PKS0823-223 & $\begin{array}{l}\text { ? abs. } \\
\text { Na D abs. }\end{array}$ & 5893 & $\begin{array}{l}5349 \\
5896\end{array}$ & ---- & Possible BI Iac \\
\hline PRS1258-321 & $N a-D a b s$. & 5893 & 5996 & 0.0175 & $\begin{array}{l}\text { Galaxy } \\
\text { cz=5300 + }=200 \mathrm{~km} / \mathrm{s}\end{array}$ \\
\hline PKS1342-314 & $\begin{array}{l}\text { G band } \\
\text { Mg I abs. } \\
\text { Na D abs. }\end{array}$ & $\begin{array}{l}4300 \\
5175 \\
5893\end{array}$ & $\begin{array}{l}4915 \\
5915 \\
5914\end{array}$ & $\begin{array}{l}0.143 \\
0.143 \\
0.144\end{array}$ & $\begin{array}{l}\text { Galaxy } \\
z=0.143+/-0.002\end{array}$ \\
\hline PKS1424+240 & $\begin{array}{l}\text { ? abs. } \\
\text { ? abs. }\end{array}$ & & $\begin{array}{l}5950 \\
6285\end{array}$ & & Possible BL Lac \\
\hline PRS1444-301 & Na D abs. & 5893 & 5985 & 0.016 & $\begin{array}{l}\text { Galaxy } \\
c z=4660+/-200 \mathrm{~km} / \mathrm{s}\end{array}$ \\
\hline PRS1601-00 & $\begin{array}{l}\text { Mg I abs. } \\
\text { Na D abs. }\end{array}$ & $\begin{array}{l}5178 \\
5893\end{array}$ & $\begin{array}{l}5604 \\
6381\end{array}$ & $\begin{array}{l}0.0822 \\
0.0827\end{array}$ & $\begin{array}{l}\text { Galaxy, weak Ha em? } \\
z=0.0825+/-0.0005\end{array}$ \\
\hline PRs2309-416 & $\begin{array}{l}\text { Mg I abs. } \\
\mathrm{Na} D \text { abs. } \\
\text { Ha } \\
\text { [S II]bland }\end{array}$ & $\begin{array}{l}5178 \\
5893 \\
6563 \\
6716\end{array}$ & $\begin{array}{l}5652 \\
6432 \\
7186 \\
7343\end{array}$ & $\begin{array}{l}0.092 \\
0.091 \\
0.095 \\
0.093\end{array}$ & $\begin{array}{l}\text { RG with weak lines } \\
z=0.093+/-0.002\end{array}$ \\
\hline
\end{tabular}

Table 3

Sources Misidentified with Galactic stars

\begin{tabular}{ll}
\hline \multicolumn{1}{c}{ Name } & \multicolumn{1}{c}{ Name } \\
\hline PKS0658-656 & PKS1451-19 \\
PKS0759+183 & PKS1540-337 \\
PKS0853+03 & PKS1750+093 \\
PRS0858-279 & PKS1814-51 \\
PRS0903-573 & PKS1942+038 \\
PRS1326+06 & PKS2259-37 \\
PKS1328-05 & PKS2311-587 \\
PRS1342-00 & PKS2359-259 \\
PKS1437-153 & \\
\hline
\end{tabular}

Peterson, B. A., Jauncey, D. L., and Wright, A. E. 1976, Ap. J. (Letters), 207, L5.

Phillips, M. M. 1977, Ap. J., 215, 746.

Shimmins, A. J., and Bolton, J. G. 1974, Aust. J. Phys. Astrophys. Suppl., No. 32, 1.

Stone, R. P. S. 1977, Ap. J., 218, 767.

Stone, R., and Baldwin, J. 1983, M.N.R.A.S., 204, 347.

Véron-Cetty, M. P., and Véron, P. 1989, ESO Scientific Report, No. 7.

Wampler, E. J., Gaskell, C. M., Burke, W. L., and Baldwin, J. A. 1984, Ap. J., 276, 403.

White, G. L., Batty, M. J., Bunton, J. D., Brown, D. R., and Corben, J. B. 1987, M.N.R.A.S., 227, 705.

Wilkes, B. J. 1986, M.N.R.A.S., 218, 331.

Wills, D., and Lynds, R. 1978, Ap. J. Suppl., 36, 317.

Wright, A. E., Ables, J. G., and Allen, D. A. 1983, M.N.R.A.S., 205, 793 . 